\title{
CASE OF \\ POPLITEAL ANEURISM,
}

IN WHICH THE

FEMORAL ARTERY WAS TIED AFTER THE SAC HAD BURST.

BY J. D. WRIGHT,

SURGEON-MAJOR, GRENADIER GUARDS.

Communicated by SIR BENJAmin C. BRODIE, Bart.

Received May 8th.--Read June 12th, 1849.

THE accompanying case of popliteal aneurism occurred in the Hospital of the Grenadier Guards, under the immediate charge of Mr. Huthwaite, Surgeon of the 3d battalion of that regiment, in conjunction with whom I daily visited the patient. It derives its interest chiefly as one of the few cases terminating favorably, in which an operation was performed after the rupture of the sac, and consequent extravasation; also of the success of that operation after the failure of compression. It has always been a matter of question whether amputation should be performed directly after the rupture of the sac, or whether the artery should be taken up and an attempt be made to save the limb.

I therefore simply offer this case to the Society as one to be added to the list already published, in order to form some data to guide us in our practice.

Private John Maiden, Grenadier Guards, aged 37, by trade a shoemaker, of spare make but well proportioned; height five feet ten inches, habitually healthy.

March 17th, 1848. Relieved off sentry and walked to hospital reporting he had pain and swelling of the left kneejoint, which he attributed to rheumatism. On a previous guard six days prior to admission, he first felt pain in the joint, and also became aware of the existence of the popliteal 
tumour; but obtaining relief by the application of a stimulating embrocation, he disregarded it at the time. On examination, the popliteal space was found to be filled with a firm pulsating tumour, of the form and size of a large orange, the nature of which could not be doubted. The superficial veins below the knee were somewhat turgid, and the limb to the same extent was affected in a slight degree with serous infiltration. There was no febrile disorder present, and we felt assured after careful investigation that no cardiac disease existed.

Such being the features of the case, it offered, as we deemed, a suitable one to effect a cure by the method of compression on the femoral artery. With but little delay the process was commenced; preparatory to which, however, he was bled to the amount of twenty ounces, and placed upon a spare diet for a few days.

On the 21st, the fourth day after admission (in the interim the tumour not having enlarged), the pad of an Italian tourniquet was applied over the artery a little above where it enters the tendinous canal. The means employed were adequate to extinguish the pulsation of the tumour, but the pressure applied to effect this could not be borne continuously, and we were obliged to rest our only hope upon partially checking the flow of blood into the tumour. The immediate consequences of the interrupted circulation in the extremity were increased turgidity of the superficial veins, a trifling degree of fulness of the limb arising from capillary congestion, and, to the feel, augmented temperature ; at first the patient was restless, and subsequently experienced several slight paroxysms of febrile disorder, but eventually he was brought to a quiescent state. As the circulation adapted itself to its altered course the tumefaction of the limb nearly disappeared, as also the turgidity of the veins. A slight degree of erythemawas produced by the pressure of the pad, which was relieved by changing somewhat the point of resistance. Under this course of treatment the tumour appeared to diminish in volume, to feel more solid, and to lose in part its strong pulsation, and we were sanguine enough to hope for a successful 
issue to our persevering efforts ; however, on the morning of the 30th of March, the eighth day of the application of the pressure, whilst the patient was motionless, he was suddenly seized with acute pain in the knee, followed by a quick tumefaction of the parts. On examination, the popliteal tumour appeared unchanged, but an additional tumour was found on the inner side of the joint, circumscribed, flattened, and pulsating in a slight degree; it was about the size of twice that of the palm of the hand, with considerable prominence : it was evident that the coats of the aneurism had given way, and allowed blood to be effused. We at once decided to tie the femoral artery, and the operation was performed by $\mathbf{M r}$. Huthwaite, after a delay of only three hours from the time that the sac burst. The patient was subjected to the influence of chloroform, and the operation carried through its progressive stages with facility and expedition. The parts divided seemingly had not suffered from the long continued pressure. The moment of the application of the ligature, the pulsation of the tumour ceased. The limb again became tumid, and the superficial veins became more turgid, accompanied by augmented heat of the skin. These effects speedily subsided. The effused blood was gradually absorbed, and the aneurismal tumour by degrees diminished. The incised parts united partly by the adhesive process, and partly by that of granulation; the ligature separated on the twenty-sixth day after the operation.

April 9th, 1849. The tumour is now reduced two thirds of its volume, and the man has recovered the perfect use of the limb, and is doing all his duties as a soldier.

Note. August 8. Maiden still works at the shoemaker's shop in his barracks, and takes the lighter guards when it comes to his turn, as he still walks rather lame. After much exercise he suffers from cramp in the calf of the leg, and swelling of the ankle. The tumour in the ham has diminished since April, and is now about the size of half a walnut; the circulation in the leg appears good. 